\title{
Optimal achromatic wave retarders using two birefringent wave plates: reply
}

\author{
Jose Luis Vilas, ${ }^{*}$ Luis Miguel Sanchez-Brea, and Eusebio Bernabeu \\ Optics Department, Applied Optics Complutense Group, Universidad Complutense de Madrid, \\ Facultad de Ciencias Físicas, Ciudad Universitaria s.n., Madrid 28040, Spain \\ *Corresponding author: jlvilas@ucm.es \\ Received 5 August 2013; accepted 3 September 2013; \\ posted 16 September 2013 (Doc. ID 195218); published 3 October 2013
}

\begin{abstract}
This reply attempts to cast some more light on the achromatic systems composed by wave plates, in particular to the calculus of the overall retardation and the use of the Jones matrix equivalence theorem. An equivalent expression for the overall retardation of the system in terms of the trace is also given. (C) 2013 Optical Society of America

OCIS codes: (220.4830) Systems design; (260.5430) Polarization; (260.1440) Birefringence; (160.1190) Anisotropic optical materials.

http://dx.doi.org/10.1364/AO.52.007081
\end{abstract}

\section{Introduction}

This article is a reply to comment of Zhang [1] about our previous article [2]. First of all, we would like to express our gratitude to the author for his interest in our work. In our paper [2], the main objective was the design of achromatic retarders using two wave plates with different birefringence. The optimization of this achromatic retarder was performed with a merit function that measured the distance between the overall retardation and the retardation target, in our case $\pi / 2 \mathrm{rad}$. In [1], Zhang shows the correct way to calculate the retardation using the Jones equivalence theorem [3].

For the optimization we considered that the angle between the fast axis of the wave plates was $\phi=0$ or $\phi=\pi / 2 \mathrm{rad}$. For this particular case, the overall retardation calculated with Eq. (11) in Ref. [2] is correct and the achromatic wave retarder obtained is valid. It is noteworthy that the condition $\phi=0$ or $\phi=\pi / 2$ must be imposed, instead of derived, as we show.

Our work is based on Saha et al. [4]. In that article, the authors calculate the overall retardation and the azimuth by equaling the matrix product

$1559-128 X / 13 / 297081-02 \$ 15.00 / 0$

(C) 2013 Optical Society of America of the two retarders with the matrix of only one wave plate,

$$
U=\left(\begin{array}{cc}
A & B \\
-B^{*} & A^{*}
\end{array}\right)=C(\Delta, \Psi),
$$

where

$$
\begin{aligned}
& C(\Delta, \Psi) \\
& \quad=\left(\begin{array}{cc}
\cos \frac{\Delta}{2}+i \sin \frac{\Delta}{2} \cos 2 \Psi & i \sin \frac{\Delta}{2} \sin 2 \Psi \\
i \sin \frac{\Delta}{2} \sin 2 \Psi & \cos \frac{\Delta}{2}-i \sin \frac{\Delta}{2} \cos 2 \Psi
\end{array}\right) .
\end{aligned}
$$

Equaling term to term, the relations obtained are

$$
\begin{gathered}
\tan ^{2} \frac{\Delta}{2}=\frac{|\operatorname{Im}(A)|^{2}+|\operatorname{Im}(B)|^{2}}{|\operatorname{Re}(A)|^{2}+|\operatorname{Re}(B)|^{2}}, \\
\tan 2 \Psi=\frac{B-B^{*}}{A-A^{*}} .
\end{gathered}
$$

Nevertheless, a system composed of several linear retarders is not, in general, equivalent to a single wave plate, as proposed by [4]. Thus, the overall retardation and the azimuth of Eqs. (3) and (4) cannot be obtained under this hypothesis. Despite this fact, 
Eq. (3) is right, but Eq. (4) is wrong. For that reason, we tried to obtain the overall retardation via diagonalization of the matrix product [2]. However, the diagonalization is valid only when $\phi=0$ or $\phi=\pi / 2 \mathrm{rad}$, since the matrix product is diagonal. Nevertheless, in a general treatment the overall retardation must be calculated using the Jones equivalence theorem, as it is pointed by Zhang [1].

\section{Additional Comments on the Calculus with the Equivalence Theorem}

We show several additional comments about our article [2] and the calculus of the overall retardation and the azimuth, $\Psi$. Equation (3) can be obtained using the Jones matrix equivalence theorem:

$$
U=R(\omega) C(\Delta, \Psi),
$$

where

$$
R(\omega)=\left(\begin{array}{cc}
\cos \omega & -\sin \omega \\
\sin \omega & \cos \omega
\end{array}\right)
$$

In addition, the Jones equivalence theorem allows us to derive another form for the expression in Eq. (3) in terms of the trace of $U$ :

$$
\cos \frac{\Delta}{2}=\frac{\operatorname{Tr} U}{2 \cos \omega} .
$$

This equation is the correct version of Relation (11) shown in [2]. However, Eq. (7) is valid for any value of the azimuth $\phi$. The equation obtained by diagonalization of matrix $U$ in Ref. [2] does not take into account the effect of the rotator, and, therefore, the term $\cos \omega$ did not appear. Thus, that expressionEq. (11) in Ref. [2] -is valid only when $\omega=0$ [2]. Despite this fact, the retarder proposed in our article is valid, due to $\omega=0$ because $\phi=0$ or $\phi=\pi / 2 \mathrm{rad}$, and, thereby, the achromatic wave retarder obtained is not affected. However, we would clarify that our retarder is optimal in thickness under the condition $\phi=0$ or $\phi=\pi / 2$; if other azimuths are considered, then we can obtain better achromatic retarders.

The azimuth and the angle of the rotator calculated from Eq. (5) are

$$
\tan 2 \Psi=\frac{\operatorname{Re}(A) \operatorname{Im}(B)+\operatorname{Re}(B) \operatorname{Im}(A)}{\operatorname{Re}(A) \operatorname{Im}(A)-\operatorname{Re}(B) \operatorname{Im}(B)},
$$

$$
\tan \omega=-\frac{\operatorname{Re}(B)}{\operatorname{Re}(A)} .
$$

Equation (8) shows the right calculus for the azimuth, $\Psi$, and must be derived from the Jones matrix equivalence theorem, instead of equaling the product matrix with a matrix of a wave plate, as proposed by Saha et al. [4]. As an example, let us consider the system formed by two wave plates with retardations $\delta_{1}=\pi / 2, \delta_{2}=3 \pi / 4$ and $\phi=\pi / 3 \mathrm{rad}$ in order to establish a comparison between Eqs. (4) and (8). Using Eq. (8) we obtain $\Psi=-0.3569 \mathrm{rad}$, while when Eq. (4) is used the azimuth is $\Psi=-0.7360 \mathrm{rad}$. This significant difference is because Eq. ( $\underline{4}$ ) does not take into account the rotator effect.

\section{Conclusions}

The Jones equivalence theorem shows how a system consisting of a set of linear retarders and rotators is equivalent to a system composed by only two elements, a rotator and a linear retarder. The application of this theorem to a system formed by two wave plates allows us to calculate the overall retardation [2]. Using this theorem, a correction to the overall retardation has been performed in terms of the trace of matrix $U$. Despite this fact, the achromatic quarter retarder proposed in [2] is valid because the rotation effect does not appear when $\phi=0$ or $\phi=\pi / 2 \mathrm{rad}$. Also, in this article we show how the expression of the azimuth obtained by Saha et al. is calculated without taking into account the effect of the rotator. Finally, the right calculus is shown.

We assume the error committed in [2] and we would like to offer our apologies to the authors of [4] and [5]. Finally we would like express our gratitude to Dr. Zhang for his comments.

\section{References}

1. X. Zhang, "Optimal achromatic wave retarders using two birefringent wave plates: comment," Appl. Opt. 52, 7078-7080 (2013).

2. J. L. Vilas, L. M. Sanchez-Brea, and E. Bernabeu, "Optimal achromatic wave retarders using two birefringent wave plates," Appl. Opt. 52, 1892-1896 (2013).

3. H. Hurwitz and R. C. Jones, "A new calculus for the treatment of optical systems. II. Proof of three general equivalence theorems," J. Opt. Soc. Am. 31, 493-495 (1941).

4. A. Saha, K. Bhattacharya, and A. K. Chakraborty, "Achromatic quarter-wave plate using crystalline quartz," Appl. Opt. 51, 1976-1980 (2012).

5. G. Kang, Q. Tan, X. Wang, and G. Jin, "Achromatic phase retarder applied to MWIR \& LWIR dual-band," Opt. Express 18, 1695-1703 (2010). 